\title{
The cutaneous microbiome in hospitalized patients with pressure ulcers
}

\author{
Luuk A. de Wert ${ }^{1,2}$, Sander S. Rensen ${ }^{1,2}$, Zita Soons $\mathbb{1}^{1,2^{*}}$, Martijn Poeze ${ }^{1,2}$, Nicole D. Bouvy ${ }^{1,2}$ \\ \& John Penders $\mathbb{D}^{2,3,4}$
}

This study investigated whether there are differences in the composition of the cutaneous microbiome of the unaffected skin between patients with pressure ulcers compared with those without pressure ulcers. The cutaneous microbiome of the unaffected skin of 15 patients with sacral pressure ulcers compared to 15 patients without pressure ulcers was analysed. It demonstrated that the inter-individual variation in skin microbiota of patients with pressure ulcers was significantly higher $(P=0.01)$. The abundance of 23 species was significantly different with Staphylococcus aureus and unclassified Enterococcus the most abundant species in patients with pressure ulcers. Random Forest models showed that eight species were associated with pressure ulcers occurrence in $81 \%$ of the patients. A subset of four species gave the strongest interaction. The presence of unclassified Enterococcus had the highest association with pressure ulcer occurrence. This study is the first to demonstrate that the cutaneous microbiome is altered in patients with pressure ulcers.

Pressure ulcers, or decubitus, are an important clinical problem, especially in the elderly, disabled, or those who are bound to a bed or wheelchair. Yearly, over 2.5 million hospitalized patients are treated in the United States for pressure ulcers with estimated costs of 11.0 billion U.S. Dollars ${ }^{1,2}$. Pressure ulcers develop as a result of prolonged mechanical loading on the skin over a bony prominence, mostly at the sacral area. Several internal factors can further contribute to the development of pressure ulcers. For example, increasing age, unconsciousness, urinary and/or bowel incontinence, poor nutritional status, Diabetes Mellitus, paralysis, and cardiovascular diseases have been associated with an increased risk of developing pressure ulcers ${ }^{3}$.

The human skin harbours a large number of microorganisms, the so-called microbiota including bacteria and fungi. A well-balanced cutaneous microbiota is essential in maintaining a healthy skin environment. Indeed, many skin diseases are associated with changes in microbiota composition. For example, an increase in relative Staphylococcus aureus abundance plays an important role in the pathophysiology of atopic dermatitis flares, while certain strains of Propionibacterium acnes contribute to the development of Acne Vulgaris ${ }^{4}$. In wounds, bacteria impair healing by forming a biofilm, which eventually, may lead to chronic non-healing wound $\mathrm{s}^{5,6}$.

In view of the impact of skin bacteria on the development of skin disorders as highlighted above, cutaneous microbiota differences may significantly contribute to the risk of developing pressure ulcers. Such potential differences may offer a new way to identify patients at increased risk for pressure ulcers and can lead to new preventive measures based on modulation of the microbiota as well ${ }^{7}$.

Therefore, the aim of this study was to examine the cutaneous microbiome of the unaffected skin of hospitalized patients with and without sacral pressure ulcers.

\footnotetext{
${ }^{1}$ Department of Surgery, Maastricht University Medical Centre, Maastricht, The Netherlands. ${ }^{2}$ NUTRIM School for Nutrition and Translational Metabolism, Maastricht University, Maastricht, The Netherlands. ${ }^{3}$ Department of Medical Microbiology, Maastricht University Medical Centre, Maastricht, The Netherlands. ${ }^{4}$ CAPHRI School for Public Health and Primary care, Maastricht University Medical Centre, Maastricht, The Netherlands. *email: zita. soons@maastrichtuniversity.nl
} 


\begin{tabular}{|l|l|l|}
\hline & $\begin{array}{l}\text { DC group } \\
(\mathbf{n = 1 5})\end{array}$ & NoDC (n=15) \\
\hline Age- years & $72.8 \pm 8.8$ & $73.2 \pm 5.5$ \\
\hline Male sex & $9(60.0)$ & $9(60.0)$ \\
\hline Height- centimetres & $172.4 \pm 9.2$ & $169.9 \pm 13.5$ \\
\hline Weight- kilogram & $78.8 \pm 20.0$ & $76.0 \pm 12.6$ \\
\hline BMI- kg/cm ${ }^{2}$ & $26.2 \pm 5.5$ & $26.7 \pm 4.0$ \\
\hline Antibiotics use & $9(60.0)$ & $8(53.3)$ \\
\hline Diagnosis of hospital admission & $7(46.7)$ & $6(40.0)$ \\
\hline Orthopaedic & $4(26.7)$ & $6(40.0)$ \\
\hline Gastro-intestinal & $3(20.0)$ & $2(13.3)$ \\
\hline Cardiovascular & $1(6.7)$ & $1(6.7)$ \\
\hline Respiratory & \multicolumn{2}{|l}{} \\
\hline Comorbidities & $3(20.0)$ & $3(20.0)$ \\
\hline Diabetes Mellitus & $1(6.7)$ & 0 \\
\hline Neuropathy & $1(6.7)$ & 0 \\
\hline Paraplegia & &
\end{tabular}

Table 1. Baseline characteristics of included participants $(n=30) *$. Data are presented as mean \pm SD or number (\%). NS = Not significant. *This number includes the samples of three patients in the control group, which failed during sequencing because of low bacterial DNA yield and which were removed from further analysis.

\section{Results}

Study population. Thirty patients were included in this study between July 2015 and August 2015, 15 patients with pressure ulcers (DC group) and 15 control patients (NoDC group). All patients were bound to a bed or chair and admitted to one of the wards of MUMC+. Patients were matched for age, sex, BMI, Diabetes Mellitus, antibiotics use, and medical diagnosis. Baseline characteristics are presented in Table 1. We obtained skin swabs from the intact skin of vertebrae level L3, and not the pressure ulcer in the sacral area itself.

Sequencing and taxonomic composition. A total of 5,021,668 paired-end reads were generated. After trimming, quality filtering, and removal of potential chimeric reads, 3,707,991 sequences were retained for downstream analysis and clustered into 2,169 operational taxonomic units (OTUs). Samples from three control participants failed during sequencing, because of low bacterial DNA yield, and were discarded for subsequent analysis. The number of sequences for the remaining samples ranged from 48,952 to 259,953 (median 117,362).

Taxonomic composition. In both patients groups, Firmicutes, Proteobacteria and Actinobacteria were the most abundant bacterial phyla, whereas at the genus-level, Staphylococcus spp. and Corynebacterium spp. predominated (Fig. 1A,B).

Microbial richness and diversity are not altered in patients with sacral pressure ulcers. The DC and NoDC groups did not show a significant difference with respect to average number of observed OTUs (DC group: 245.0 (168.0-370.0)), NoDC group 299.5 (163.0-478.0)) (Fig. 2A). The estimated richness of the DC group (Chao1, 299.6 (195.1-1112)) and No DC group (Chao1, 287.8 (204.0-504.3)) did not differ significantly (Fig. 2B). The Good's estimator suggested $>99.95 \%$ coverage for all samples included in the present study, indicating that only an additional five OTUs would have been found if the sequencing depth were increased with 1,000 reads. Also, microbial diversity as estimated by the Shannon diversity index was not statistically significantly different in skin swabs between the DC and NoDC group, although a larger variation in microbial diversity was observed in the DC group (Fig. 2C). Altogether these results indicate that the microbial richness and evenness are not affected in patients with sacral pressure ulcers.

The skin microbial community structure is related to disease occurrence. Next, we assessed the dissimilarity in the microbial composition (beta-diversity) of the skin using the Bray-Curtis and the weighted Unifrac distances. Unifrac distances are based on the fraction of branch length shared between two communities within a phylogenetic tree constructed form the $16 \mathrm{~S}$ rRNA gene sequences from all communities being compared. A relatively small UniFrac distance implies that two communities are compositionally similar, harbouring lineages sharing a common evolutionary history ${ }^{8}$.

Visualization of Bray-Curtis and weighted Unifrac dissimilarities using PCoA, indicated that many, but not all patients in the DC group clustered apart from the NoDC group patients (Fig. 3A,C). Separation was statistically significant as tested by permutational multivariate analysis of variance on these distance metrics $(\mathrm{p}=0.008$ and $\mathrm{p}<0.001$, respectively). This implies that the skin microbial communities in part of the patients with sacral pressure ulcers are structurally and significantly different from the microbial communities in patients without pressure ulcers.

Moreover, the dissimilarity in the microbial community structures in skin swabs within the DC group was significantly larger than the dissimilarity within the NoDC group. This indicates that the inter-individual variation of the skin microbiota of patients without sacral pressure ulcers is significantly smaller as compared to the 

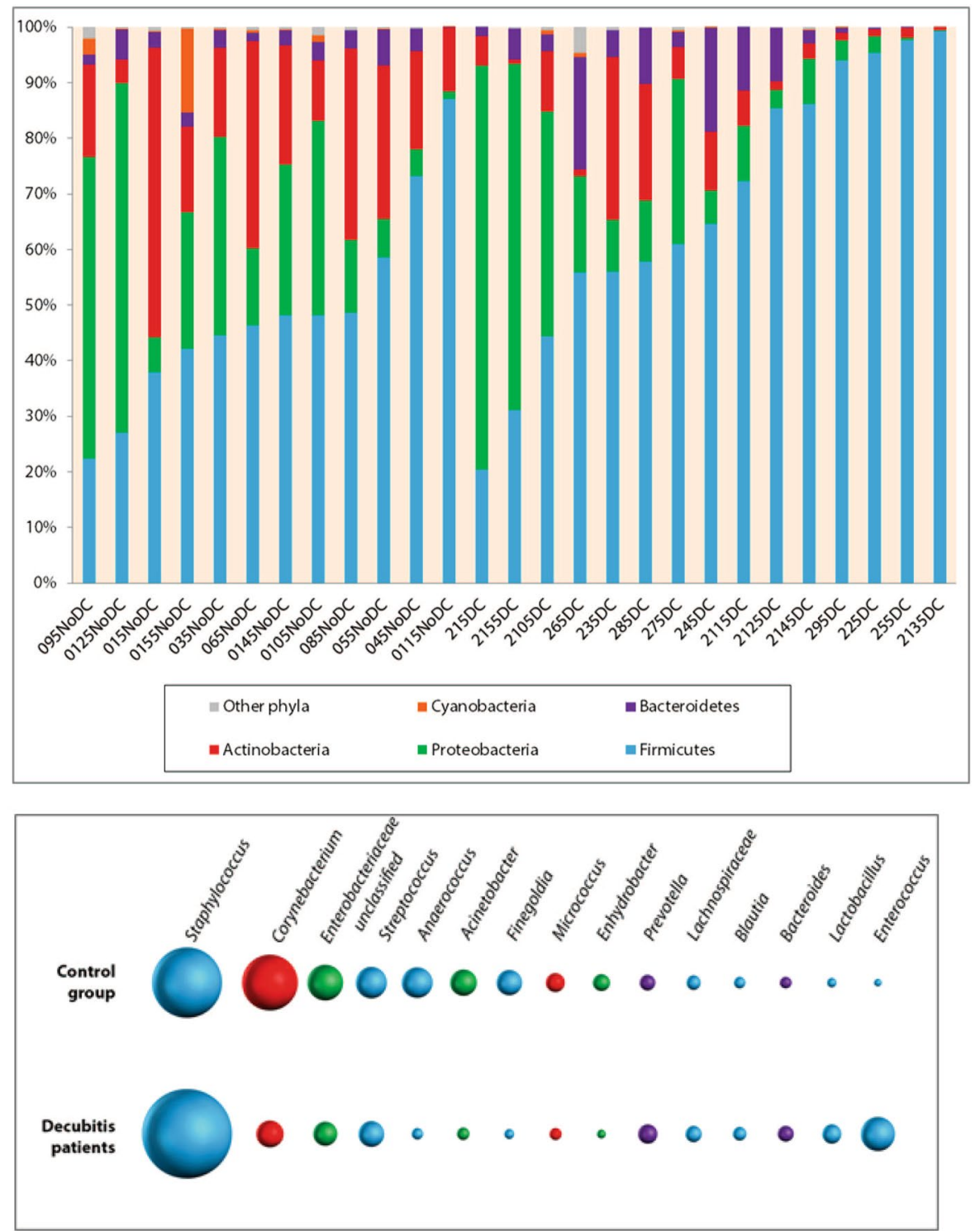

Figure 1. (A) Relative abundance of the most predominant bacterial phyla in the control (NoDC) and decubitus group (DC) patients. (B) Average proportion of the 15 most abundant bacterial genera in the control group (NoDC) and decubitus group (DC) patients.
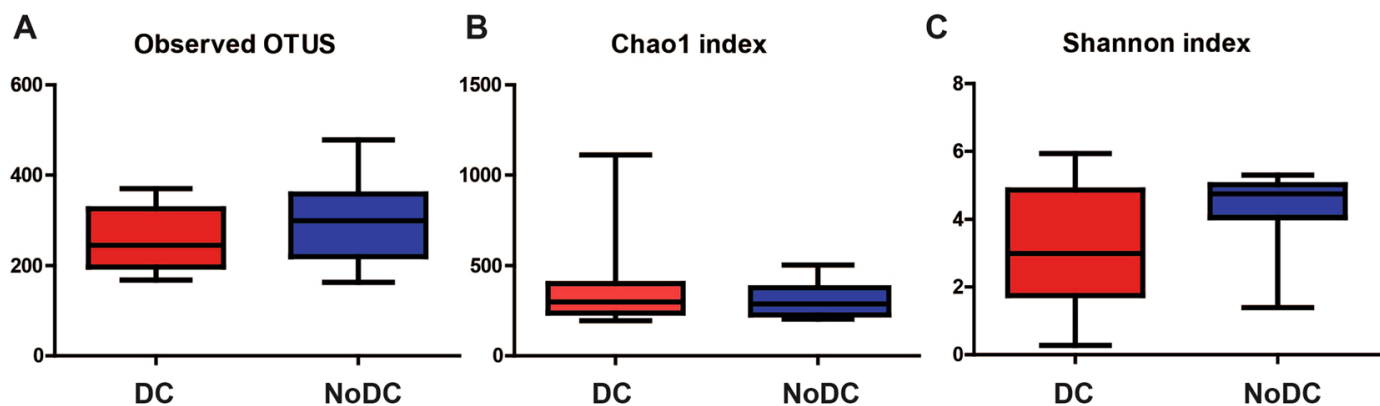

Figure 2. Box and Whisker plots of the Alpha diversity metrics. (A) Observed OTUs, $P>0.05$ Mann Whitney U test. (B) Chaol index, P > 0.05 Mann Whitney U test. (C) Shannon index, P >0.05 Mann Whitney U test. 

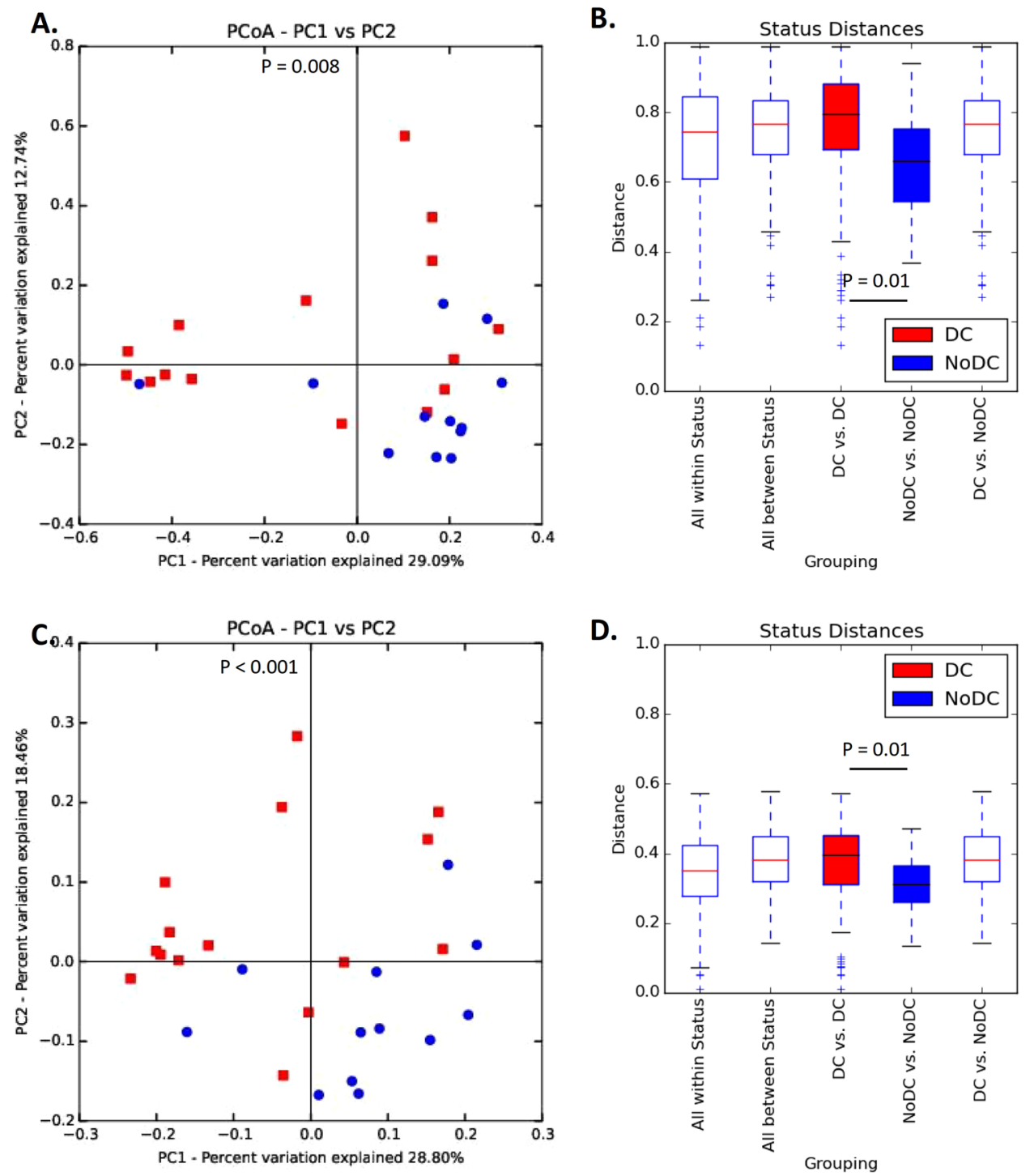

Figure 3. Microbial community structure (at OTU-level) for patients with and without sacral pressure ulcers. (A) Principal Coordinate Analyses (PCoAs) based on Bray-Curtis distance coloured according to disease occurrence (DC group patients in red and NoDC group patients in blue). Variation explained by the principal coordinates: PC1 (29.1\%); PC2 (12.7\%). (B) Box and Whisker plots of the within and between group BrayCurtis distances. (C) Principal Coordinate Analyses (PCoAs) based on weighted Unifrac distance coloured according to disease occurrence (decubitus group patients in red and control group patients in blue). Variation explained by the principal coordinates: PC1 (28.8\%); PC2 (18.5\%). (D) Box and Whisker plots of the within and between group weighted Unifrac distances.

inter-individual variation in the cutaneous microbiome of patients with sacral pressure ulcers (Fig. 3B, $\mathrm{P}=0.01$ for Bray-Curtis and Fig. 3D, $\mathrm{P}=0.01$ for weighted Unifrac).

Species abundance. Hierarchical clustering based upon the species level relative abundance revealed distinct clusters for half of the patients with and without pressure ulcers (Fig. 4A). A few patients in the DC group were closer to disease free patients of the NoDC group based on the overall species abundancies. We next focussed on the species that distinguish patients with and without pressure ulcers (DC and NoDC group). The abundance of 23 species was significantly different, ranging from a 184-fold more abundant presence (log2 fold change of 7.5) to a 34-fold less abundant presence (log2 fold-change of 5.1) in the DC group. Staphylococcus aureus and unclassified Enterococcus were the most abundant species in the DC group. (Fig. 4B,C). Unclassified Corynebacterium and unclassified Streptococcus were also very prevalent in the dataset, but did not show differential abundance. Microbiome abundance tables contain many zeros (Supplementary File 1, Fig. 2). The median abundance of a species is zero, the mean $\log 2$ abundance 2.1 . The prevalence of any of the 23 differentially abundant (DA) species is thus above average. In particular the ten highest ranked DA species based on p-values are highly abundant. The DA species with $0.01<$ p-value $<0.05$ are low-abundant ones. Despite the low abundance 
A.

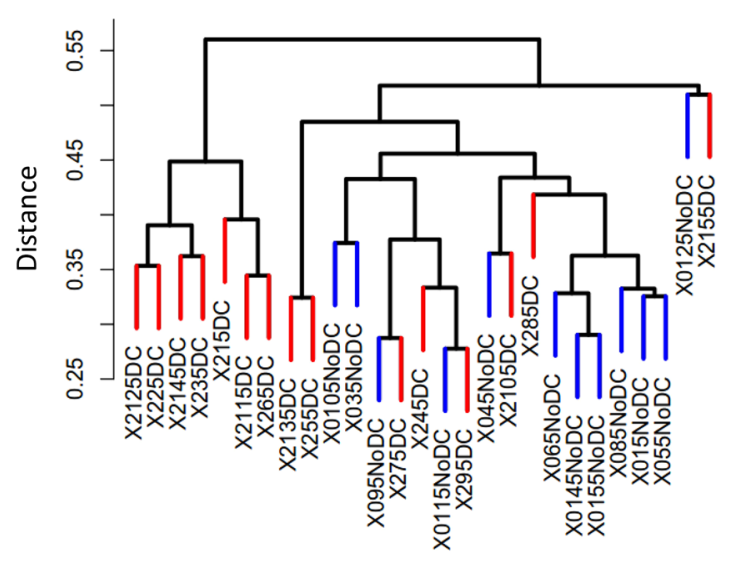

C.
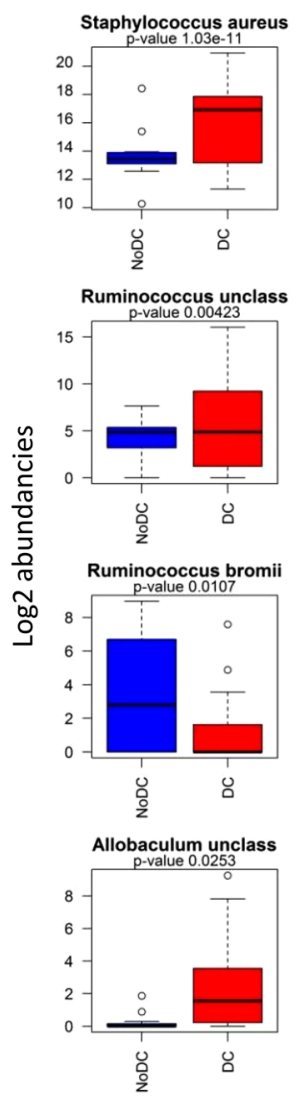
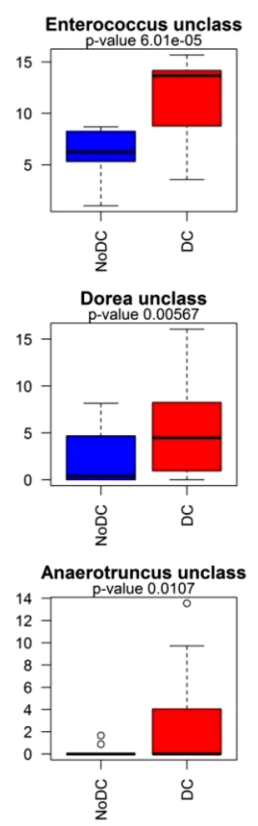

Christensenella unclass

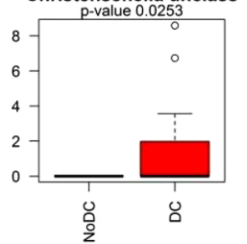

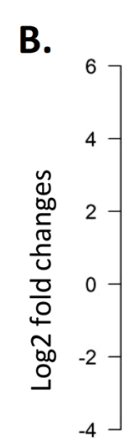
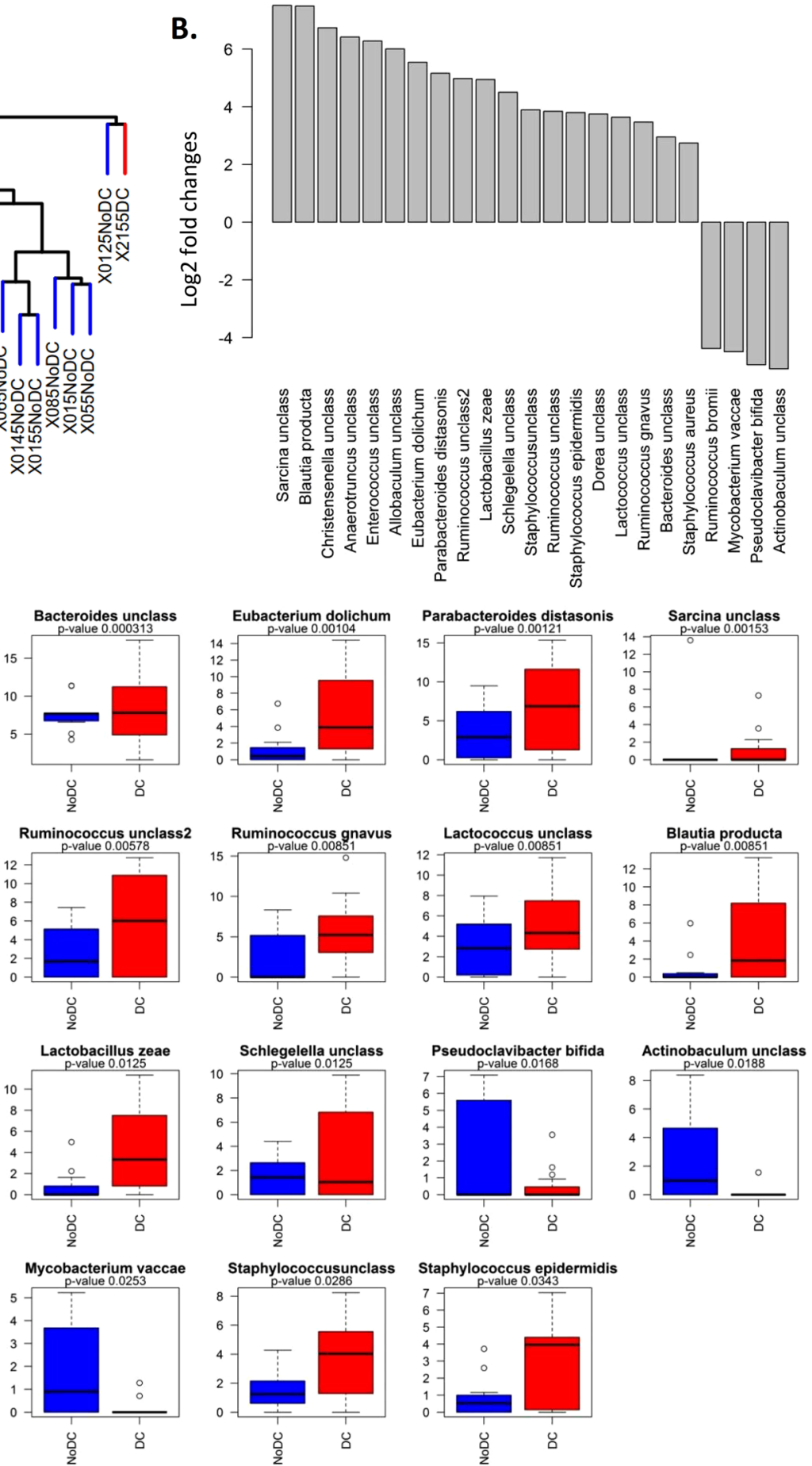

Staphylococcus epidermidis

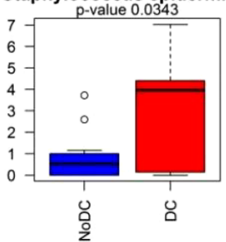

Figure 4. Differential species abundancies between DC and NoDC group patients. (A) Cluster diagram. (B) $\log 2$ fold changes of the species with significantly different abundance in DC vs. NoDC group patients. (C) Boxplots of $\log 2$ abundancies of these 23 species.

on average, the presence of these species in certain patients might be distinct between DC or noDC, which we investigate using random forests.

Random forest analysis. To further test the potential clinical relevance of bacterial species and clinical factors in pressure ulcers, we carried out a Random Forest analysis using the species abundances, resp. clinical data. We found that the clinical data in Table 1, such as sex, antibiotics use, and BMI, were not significantly related to pressure ulcer occurrence. Supplementary Figure 3 (Supplementary File 1) shows that none of the clinical factors has a higher correlation to pressure ulcer outcome than the best random predictor. In addition, when all clinical 


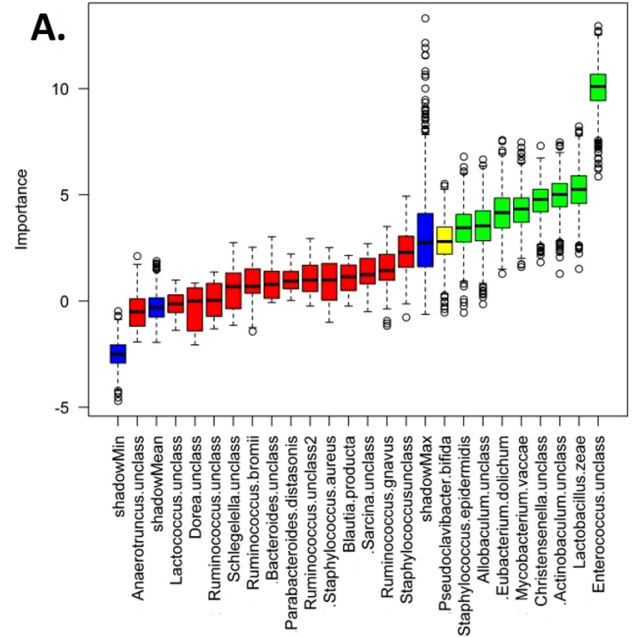

C.

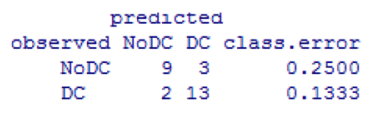

Overall error rate: 18.52 용
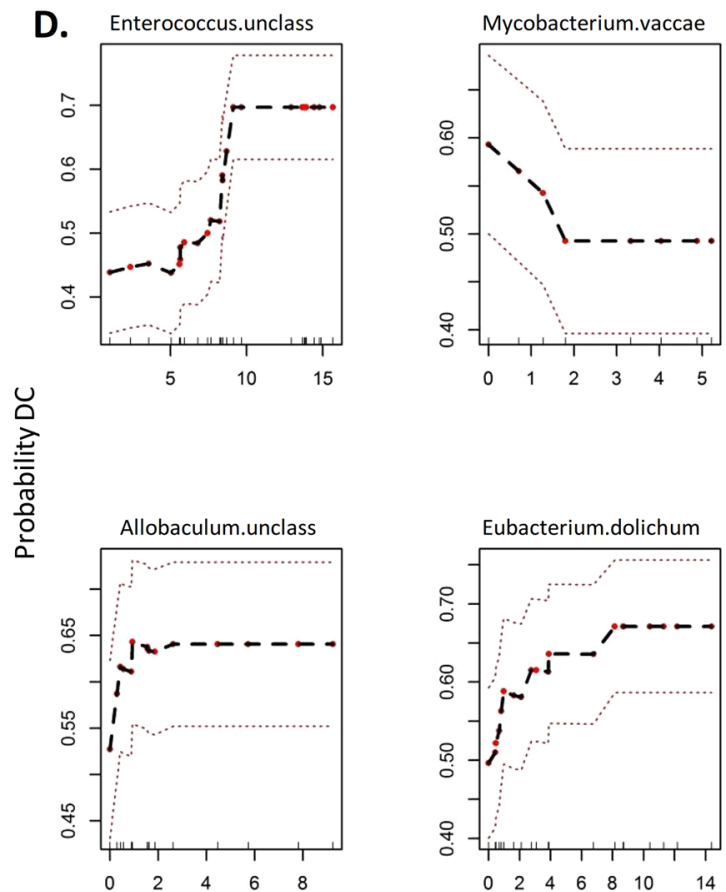

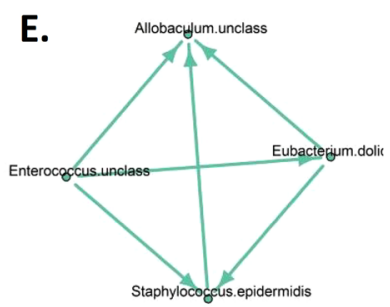

B. all

Enterococcus.unclass Lactobacillus.zeae

Eubacterium.dolichum

Mycobacterium.vaccae

Actinobaculum. unclass

Staphylococcus.epidermidis

Allobaculum.unclass

Christensenella.unclass

NoDC

Enterococcus.unclass

Lactobacillus.zeae

Eubacterium dolichum

Mycobacterium.vaccae

Actinobaculum.unclass

Staphylococcus.epidermidis

Allobaculum.unclass

Christensenella.unclass

$$
\text { DC }
$$

Enterococcus.unclass

Lactobacillus.zeae

Eubacterium.dolichum

Mycobacterium.vaccae

Actinobaculum unclass

Staphylococcus.epidermidis

Allobaculum.unclass

Christensenella.unclass

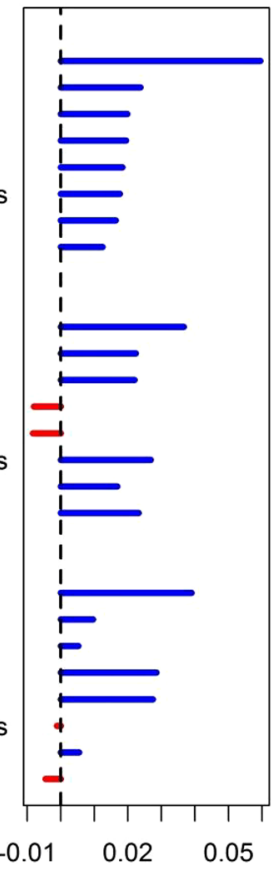

Variable Importance: cl
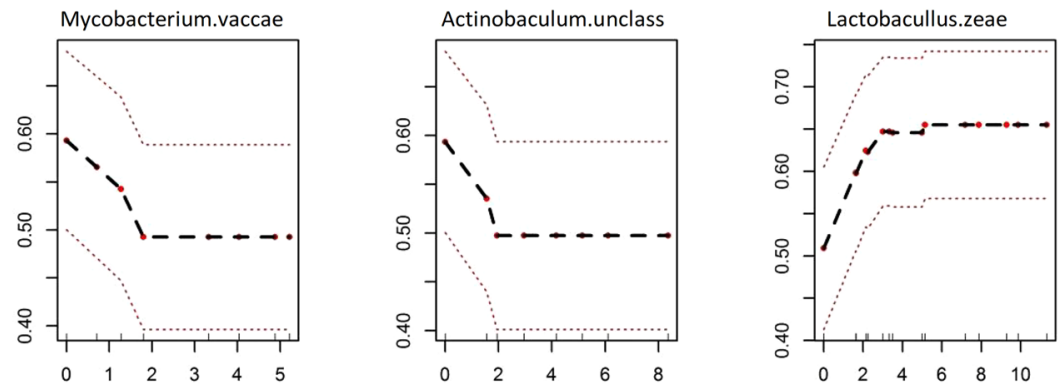
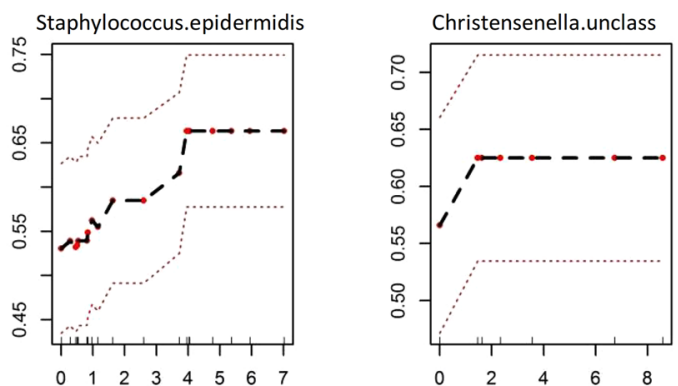

Log2 abundancies

Figure 5. Random Forests with Boruta feature selection using species abundance data. (A) The importance of each feature across 1000 repeats is shown compared with the worst, best, and mean randomly generated features. (B) Variable importance in the random forest. (C) Confusion matrix. (D) Effect of species abundance on expected pressure ulcer occurrence in the random forest model. Values on the vertical axis represent the expected probability of a pressure ulcer for a given species, after adjusting for all other predictors. Dashed red lines represent \pm 2 standard error. The red dots represent samples with corresponding inner lines on the $\mathrm{x}$-axis representing the values of the abundancies. Expected probability based on random classification is 0.44 . (E) Interaction plot. 
data were taken together, the observed error rate (41\%) is not significantly better either than a random predictor in a Fisher test $(50 \%)$.

Classification based on species abundance, however, showed that eight species are significantly related to pressure ulcer occurrence in the Boruta algorithm (Fig. 5A). These eight species together classified pressure ulcer occurrence correctly in $81 \%$ of the patients (error rate of 19\%) (Fig. 5C). Figure 5B shows that unclassified Enterococcus is also the most important variable for classification as DC and NoDC in a multivariate model. Figure 5D shows the expected probability of having a pressure ulcer as function of species abundance, adjusted for all other species abundances. High abundance of for instance, unclassified Actinobaculum is negatively associated with a higher probability of having a pressure ulcer, whereas the abundance of unclassified Enterococcus is positively associated with higher probability of having a pressure ulcer.

Interestingly, a subset of four species giving the strongest interactions in an interaction plot (Fig. 5E), unclassified Enterococcus, unclassified Allobaculum, Eubacterium dolichum and Staphylococcus epidermidis, correctly classify pressure ulcer occurrence in $74 \%$ of the patients (error rate of $26 \%$ ). Species tending to split close to the root node have a strong effect on classification, whereas species that split further down the tree have less impact on pressure ulcer occurrence. Species that split close to each other in the tree represent stronger interactions, and thus together have greater impact on pressure ulcer occurrence. The source of the arrow in the interaction plot is the more influential species (split closer to the root node). More specifically, the presence of unclassified Enterococcus has the highest impact on pressure ulcer occurrence. Since unclassified Enterococcus, unclassified Allobaculum, Eubacterium dolichum and Staphylococcus epidermidis are highly abundant in different patients, a combination of these four species contains additive information leading to better classification of pressure ulcer occurrence.

\section{Discussion}

The cutaneous microbiome plays an important role in providing a healthy environment of the skin, and microbiome alterations influence host-microbe interactions leading to changes in host metabolism and immunity ${ }^{9}$. Therefore, maintaining a well-balanced cutaneous microbiome is essential in preventing the development of skin diseases. To our knowledge, this study is the first to investigate whether there are differences in the cutaneous microbiome of the unaffected skin between hospitalized patients with pressure ulcers compared with those without pressure ulcers. It demonstrates that the microbiota of the unaffected skin in patients with sacral pressure ulcers differs from those without pressure ulcers. Although microbial diversity and richness were not different between patients with or without sacral pressure ulcers, there was an increase in inter-individual variation of the cutaneous microbiome in patients with sacral pressure ulcers. In addition, the abundance of 23 bacterial species on the skin were significantly different in patients with sacral pressure ulcers.

The development of pressure ulcers is the result of prolonged mechanical loading in the form of pressure and shear forces. However, local changes in the skin microclimate have been shown to be a marked risk factor for the development of pressure ulcers in earlier studies ${ }^{10-12}$. In addition, changes in environmental factors influence the skin microenvironment and lead to differences in the composition of the cutaneous microbiome ${ }^{13}$. Cell damage caused by mechanical loading triggers an immune response by the release of damage associated molecular patterns (DAMPs) to initiate the process of cell repair. Several studies have shown that the cutaneous microbiota acts as a modulator of this immune response by the production of metabolites or pathogen associated molecular patterns. For example, a mouse study demonstrated that mice treated with oral vancomycin display cutaneous microbiota dysbiosis and downregulation of RegIIIy and IL-17, leading to delayed wound healing ${ }^{14}$. It is unlikely that changes in the microbiota directly cause pressure ulcers to develop, but they may play a role in the repair of mechanically damaged skin cells. Possibly, the microbiota influences the ability to recover from mechanically induced skin damage and therefore could be related to pressure ulcer development. Based on the results of the present study we only demonstrate association and cannot draw conclusions about causality.

It is particularly promising that we were able to identify eight species that were associated with pressure ulcer occurrence in random forest models. A high abundance of one of defined six species was associated with a higher prevalence of sacral pressure ulcers, suggesting that patients with a higher abundance of one of these species might already be at higher risk for developing sacral pressure ulcers. Combining information on the abundance of several species further improved the classification of the presence of a pressure ulcer. Therefore, assessing pressure ulcer correlates based on a multivariable approach with microbial species as biomarkers might provide a potential novel method to pressure ulcer risk assessment in hospitalized patients in the future.

An analysis of wound microbiota performed by Wolcott and colleagues ${ }^{6}$ demonstrated a high abundance of Staphylococcus aureus, Staphylococcus epidermidis, and Pseudomonas aeruginosa in pressure ulcers. It must be noted that some changes in the cutaneous microbiota could also be the result of a pressure ulcer near the sampling site. Indeed, we demonstrated a high abundance of Staphylococcus aureus and Staphylococcus epidermidis in our study. However, both species were not associated with pressure ulcer occurrence in our random forest models, so it is most likely that other factors were responsible for the shift in microbiota species.

Interestingly, unclassified Enterococcus and Eubacterium dolichium, both commensal gut bacteria, contribute most to the random forest model. Furthermore, a higher abundance of several other gut bacteria including Christensenella, Lactobacillus zeae and unclassified Allobaculum were also associated with a higher occurrence of pressure ulcers. In hospitalized patients, up to $17 \%$ have some form of faecal incontinence and it is a daily challenge for healthcare workers to prevent faecal incontinence in patients ${ }^{15}$. Although faecal incontinence is associated with an increased pressure ulcer risk ${ }^{16,17}$, there are also studies with contradictory results ${ }^{18,19}$. However, based on the results of the present study, it could be beneficial to protect the skin against faecal incontinence to prevent the development of pressure ulcers. 
On the other hand, a higher abundance of four species was found in patients without pressure ulcers, where two species in particular, unclassified Actinobaculum and Myobacterium vaccae, were associated with absence of pressure ulcers in the random forest models. Interestingly, Keshavarz Valian and colleagues ${ }^{20}$ demonstrated that the administration of a low dose of Myobacterium vaccae protected mice against the development of skin ulcera caused by a parasitic infection with Leishmania major. Therefore, there might be a role of specific types of bacteria in protecting the skin against the development of wounds such as pressure ulcers in humans as well.

Immobile patients are at increased risk for pressure ulcer development. All participants in our study were bound to a bed or chair and therefore at increased risk for pressure ulcer development. The cutaneous microbiome is also known to be influenced by several other clinical factors such as BMI, diabetes mellitus ${ }^{21}$, antibiotic use ${ }^{14}$, $\operatorname{sex}^{22}$, and age ${ }^{23}$. The results of our study did not reveal any relation of these clinical factors with pressure ulcers, because all patients were matched with comparable control patients. This supports that differences in outcomes in the current study were not likely to be caused by confounding factors. In order to minimize differences in hygienic procedures, patients were also matched with control participants from the same hospital wards.

Other measures to prevent external influences on outcomes included sampling by one member of the research team, sampling in similar weather conditions (July-August), and at approximately the same time every day (01.00-03.00 PM). However, many other factors could contribute to pressure ulcer development and changes in cutaneous microbiome composition, e.g. nutritional status, smoking behaviour, and vasopressor agents.

Some other comments need to be placed with respect to the interpretation of the results. First, we focussed on sacral pressure ulcers while pressure ulcers can develop at other skin areas (e.g. the heels, elbows, hips) as well. This might limit the generalizability to patients who developed pressure ulcers at different sites, because the composition of the cutaneous microbiome depends on the type of microenvironment (dry, moist, sebaceous) ${ }^{13}$. We chose to focus on sacral pressure ulcers, because those are most common in hospitalized patients ${ }^{24,25}$, and are more difficult to prevent compared to pressure ulcers at other anatomical locations.

In addition, we chose to obtain skin swabs from the skin of vertebrae level L3 and not to obtain samples from the sacral area itself, because we wanted to obtain skin samples from the unaffected skin and not from the pressure ulcers themselves. Although we did not obtain skin swabs from the sacral area, it is thought that the type of cutaneous microenvironment of vertebrae level L3 is comparable with those of the sacral area ${ }^{13}$.

Second, this study is limited by the relatively small sample size, but also its cross-sectional design. Despite the small sample size, statistically significant differences were found between the two groups. However, it is impossible to determine if these differences in cutaneous microbiome caused changes in skin physiology that eventually promoted the development of pressure ulcers, or whether the microbial shifts were a consequence of alterations in skin physiology/microenvironment as a result of prolonged mechanical loading. Although pressure ulcers are mainly caused by prolonged mechanical loading on the skin, future prospective studies should assess whether the changes in the cutaneous microbiome prior to the manifestations of pressure ulcers are a risk factor for pressure ulcer development. In addition - although random forest bootstrapping methods have been shown to be nearly identical to cross-validation - if the subcutaneous microbiome is to be used as a biomarker, validation using a larger, independent cohort is required.

Third, a limitation of the present study is the 16S rRNA (V4) hypervariable gene region that has been sequenced to profile skin microbiota. It has been shown that the V4 region is less able to capture the skin microbiota when compared to e.g. the V1-V3 region ${ }^{26}$.

In conclusion, this study is the first to demonstrate that the cutaneous microbiome of the unaffected skin differs in patients with sacral pressure ulcers from those without pressure ulcers. In the future, this might offer a novel method to improve assessment of patients at risk for development of pressure ulcers using the cutaneous microbiome as a biomarker.

\section{Methods}

Participants. We conducted a single centre, cross-sectional, case-control study at the Maastricht University Medical Centre (MUMC + ). The study was approved by the medical ethics committee of the MUMC + and written informed consent was obtained from all the study participants. The study was conducted in compliance with ethical rules for human experimentation that are stated in the Declaration of Helsinki. A total of thirty patients were able to participate at this study; 15 patients with a hospital acquired category $\geq 2$ sacral pressure ulcer (decubitus group, DC) and 15 patients with no (pre-)existing pressure ulcers (control group, NoDC). All patients had to be bound to their bed or chair admitted to one of the wards of the MUMC+.

Exclusion criteria were: age $<18$ years, mentally disabled patients/unable to give informed consent, any skin diseases/wounds/signs of infection at the skin of vertebrae level L3.

Hygiene and treatment of pressure ulcers. In our hospital, immobile patients (all study participants) were washed one time a day in the morning. This was done by bed bath using soap, towels, water, and washcloths. There was no difference in washing procedures in patients with or without pressure ulcers. Standard pressure ulcer treatment consisted of an anti-decubitus mattress and repositioning. The local treatment of pressure ulcers consisted of the application of a foam wound dressing in ten patients. Three patients had anti septic crème applied on the pressure ulcer underneath their wound dressing. Two patients had a vacuum-assisted closure system on their pressure ulcer. One patient received barrier crème between his buttocks. One patient received no extra local treatment.

Sample collection. To study the cutaneous microbiome of the unaffected skin, skin samples were obtained using Copan FloqSwabs ${ }^{\mathrm{TM}}$ (Copan Flock Technologies, Brescia, Italy) under sterile conditions. First, the skin of vertebrae level L3 was inspected for any signs of infection (erythema), skin diseases or wounds. When the skin 
was unaffected, an area of $4 \mathrm{~cm}^{2}$ was marked with a skin marker. The swab was pre-moistened with Reduced Transport Fluid (RTF) buffer ${ }^{27}$. Then, the skin was stretched and swabbed 50 times back and forth applying firm pressure. After swabbing, the tips were placed in a vial containing $50 \mu \mathrm{l}$ of RTF buffer and immediately placed on dry ice before storage in a $-80^{\circ} \mathrm{C}$ freezer until further processing. All samples were collected between 01.00-03.00 PM. To prevent bias due to variation in sample collection, all skin samples were obtained by one single researcher.

Subsequently, sex, age, length, weight, medical diagnosis, co- morbidities, antibiotics use, pressure ulcer category, and pressure ulcer treatment were recorded for each patient.

DNA isolation. After adding $180 \mu \mathrm{l}$ lysis buffer $(20 \mathrm{mM}$ Tris pH8, $2 \mathrm{mM}$ EDTA pH8, 1.2\% Triton X-100) with lysozyme $(20 \mathrm{mg} / \mathrm{ml})$, swabs were incubated for $30 \mathrm{~min}$ at $37^{\circ} \mathrm{C}$ and subsequently mixed by vortexing. After adding $200 \mu \mathrm{l}$ Buffer AL and $25 \mu \mathrm{l}$ Proteinase $\mathrm{K}$ from the QIAamp DNA mini kit (Qiagen, Hilden, Germany) and mixing by vortexing, samples were incubated overnight at $56^{\circ} \mathrm{C}$. The next day, $200 \mu \mathrm{l}$ of ethanol $(96-100 \%)$ was added and mixed by vortexing the complete lysate. Subsequent steps were conducted according to the protocol of the QIAamp DNA mini kit as per manufacturer's instructions, except that the DNA was eluted in a final volume of $100 \mu \mathrm{l}$.

Sequencing. Amplicon libraries and sequencing was performed according to previously published proto$\operatorname{cols}^{27}$. Briefly, the V4 region of the 16S rRNA gene was PCR amplified from each DNA sample in triplicate using the $515 \mathrm{f} / 806 \mathrm{r}$ primer pair $^{28}$. Pooled amplicons from the triplicate reactions were purified using AMPure XP purification (Agencourt, Massachusetts, USA) according to the manufacturer's instructions and eluted in $25 \mu 1$ $\times$ low TE $(10 \mathrm{mM}$ Tris-HCl, $0.1 \mathrm{mM}$ EDTA, pH 8.0) and subsequently quantified by Quant-iT PicoGreen dsDNA reagent kit (Invitrogen, New York, USA) using a Victor3 Multilabel Counter (Perkin Elmer, Waltham, USA). Amplicons were mixed in equimolar concentrations to ensure equal representation of each sample and sequenced on an Illumina MiSeq instrument.

Data analysis and statistics. The V4 16S rDNA bacterial sequences generated in the present study were deposited in QIITA archive (accession number: 11145). Filtering, denoising, removing of chimeric sequences, and clustering of sequences in Operational Taxonomic Units (OTUs) at 97\% similarity was conducted using the LotuS (version 1.39) pipeline ${ }^{29}$. First, sequences with an average quality below 27, read length below 170 bases, one or more ambiguous bases, or containing homopolymer stretches of over 8 bases were discarded for further analysis. Retained sequences were chimera filtered and clustered into OTUs with UPARSE ${ }^{30}$. Taxonomic annotation of OTUs was derived from RDP naïve bayes classifier annotations (minimum acceptance confidence set at 0.8$)^{31}$.

Singletons and OTUs with less than 10 reads were removed. QIIME version 1.8.1 $1^{32}$ and R version 3.1.3 were used to conduct downstream analyses.

Assessing differences on a species level. To examine potential differences in the relative abundance of bacterial species between pressure ulcer patients (DC) and control patients (NoDC), all OTUs that were taxonomically assigned to the same species were combined. We evaluated different methods for normalization and abundance testing by the R tool DAtest ${ }^{33}$ (Supplementary File 1). For all subsequent analyses, we normalized the count-table using variant stabilization by the R-package DESeq. $2^{34}$. The number of sequence reads ranged from 29,073 to 258,211 species counts in one sample. We used size factor correction to account for these differences in sequencing depth between the samples. DESeq. 2 was also applied to obtain dispersion estimates and to test for differential abundance of each species between pressure ulcer patients (DC) and control patients (NoDC) using default parameters. Results are reported as $\log 2$ fold changes and associated adjusted p-values (BH-correction).

Dendrograms were obtained by hierarchical clustering using Ward's method where Pearson's correlation was used as the distance measure.

Microbial alpha and beta diversity. The following metrics of species richness and diversity within communities (alpha-diversity) were determined: Observed OTUs (observed richness), Chao1 index (estimated richness), Shannon index (estimated diversity) and Good's coverage. Normality was tested with the D'agostino \& Pearson omnibus test. The Mann Whitney U test was used to compare the alpha diversity metrics among the patients in the DC group to the patients in the NoDC group.

The dissimilarity in the microbial community composition (beta-diversity) between each pair of skin samples was assessed using the Bray-Curtis and (un)weighted UniFrac distance ${ }^{8}$ and Bray-Curtis dissimilarity (BC). Clustering of samples was visualized using Principal Coordinate analysis (PCoA). Separation of pressure ulcer patients and control patients was tested by means of permutational multivariate analysis of variance on both the Bray-Curtis and weighted Unifrac distance matrixes using the Adonis function as implemented in the R package vegan using 1000 permutations. Nonparametric two- sample t-tests with Bonferroni correction based upon 1000 Monte Carlo permutations were used to compare the beta-diversity within pressure ulcer patients (average pairwise distance between all samples in the DC group) to the beta diversity within the control patients (NoDC) (average pairwise distance between all samples in the NoDC group) and the between group beta-diversity (average pairwise distance between samples of each patient in the DC group and each NoDC group patient).

Machine learning. Random forests are an effective approach for analyzing and interpreting high-dimensional data. The method correlates species abundance or clinical data with pressure ulcer occurrence and introduces a 
novel bivariate node-splitting. We used the $\mathrm{R}$ package randomForestSRC $\mathrm{S}^{35}$ and the Boruta algorithm for feature selection. The bootstrapped feature selection was repeated 1000 times with differing random seeds. A number of trees of 500 was found to produce a stable error rate.

Received: 10 May 2018; Accepted: 5 March 2020;

Published online: 06 April 2020

\section{References}

1. Sen, C. K. et al. Human skin wounds: a major and snowballing threat to public health and the economy. Wound repair and regeneration 17, 763-771 (2009).

2. Brem, H. et al. High cost of stage IV pressure ulcers. American journal of surgery 200, 473-477 (2010).

3. Coleman, S. et al. Patient risk factors for pressure ulcer development: systematic review. International journal of nursing studies 50, 974-1003 (2013).

4. Findley, K. \& Grice, E. A. The skin microbiome: a focus on pathogens and their association with skin disease. Plos pathogens 10, e1004436 (2014)

5. Costerton, J. W., Stewart, P. S. \& Greenberg, E. P. Bacterial biofilms: a common cause of persistent infections. Science 284, 1318-1322 (1999).

6. Wolcott, R. D. et al. Analysis of the chronic wound microbiota of 2,963 patients by $16 \mathrm{~S} \mathrm{rDNA}$ pyrosequencing. Wound repair and regeneration 24, 163-174 (2016).

7. Turnbaugh, P. J. et al. The human microbiome project. Nature 449, 804-810 (2007).

8. Lozupone, C. \& Knight, R. UniFrac: a new phylogenetic method for comparing microbial communities. Applied and environmental microbiology 71, 8228-8235 (2005).

9. Naik, S. et al. Compartmentalized control of skin immunity by resident commensals. Science 337, 1115-1119 (2012).

10. Yoshimura, M. et al. Microclimate is an independent risk factor for the development of intraoperatively acquired pressure ulcers in the park-bench position: A prospective observational study. Wound repair and regeneration 23, 939-947 (2015).

11. Yusuf, S. et al. Microclimate and development of pressure ulcers and superficial skin changes. International wound journal 12, 40-46 (2015).

12. Gefen, A. How do microclimate factors affect the risk for superficial pressure ulcers: a mathematical modeling study. Journal of tissue viability 20, 81-88 (2011).

13. Grice, E. A. \& Segre, J. A. The skin microbiome. Nature reviews microbiology 9, 244-253 (2011).

14. Zhang, M. et al. Oral antibiotic treatment induces skin microbiota dysbiosis and influences wound healing. Microbial ecology 69, 415-421 (2015).

15. Junkin, J. \& Selekof, J. L. Prevalence of incontinence and associated skin injury in the acute care inpatient. Journal of wound, ostomy, and continence nursing 34, 260-269 (2007).

16. Allman, R. M. Pressure ulcers among the elderly. The New England journal of medicine 320, 850-853 (1989).

17. Brandeis, G. H., Ooi, W. L., Hossain, M., Morris, J. N. \& Lipsitz, L. A. A longitudinal study of risk factors associated with the formation of pressure ulcers in nursing homes. Journal of the American Geriatrics Society 42, 388-393 (1994).

18. Allman, R. M., Goode, P. S., Patrick, M. M., Burst, N. \& Bartolucci, A. A. Pressure ulcer risk factors among hospitalized patients with activity limitation. Jama 273, 865-870 (1995).

19. Reed, R. L., Hepburn, K., Adelson, R., Center, B. \& McKnight, P. Low serum albumin levels, confusion, and fecal incontinence: are these risk factors for pressure ulcers in mobility-impaired hospitalized adults? Gerontology 49, 255-259 (2003).

20. Keshavarz Valian, H., Khoshabe Abdollah Kenedy, L., Nateghi Rostami, M., Miramin Mohammadi, A. \& Khamesipour, A. Role of Mycobacterium vaccae in the protection induced by first generation Leishmania vaccine against murine model of leishmaniasis. Parasitology research 103, 21-28 (2008).

21. Redel, H. et al. Quantitation and composition of cutaneous microbiota in diabetic and nondiabetic men. The Journal of infectious diseases 207, 1105-1114 (2013).

22. Fierer, N., Hamady, M., Lauber, C. L. \& Knigh, T. R. The influence of sex, handedness, and washing on the diversity of hand surface bacteria. Proceedings of the National Academy of Sciences of the United States of America 105, 17994-17999 (2008).

23. Ying, S. et al. The Influence of Age and Gender on Skin-Associated Microbial Communities in Urban and Rural Human Populations. Plos One 10, e0141842 (2015).

24. Sardo, P. M. et al. Analyses of pressure ulcer incidence in inpatient setting in a Portuguese hospital. Journal of tissue viability 25, 209-215 (2016).

25. Schoonhoven, L., Bousema, M. T., Buskens, E. \& prePURSE-study group. The prevalence and incidence of pressure ulcers in hospitalised patients in the Netherlands: a prospective inception cohort study. International journal of nursing studies 44, 927-935 (2007).

26. Meisel, J. S., Hannigan, G. D. \& Tyldsley, A. S. Skin microbiome surveys are strongly influenced by experimental design. J Invest Dermatol. 136, 947-956 (2016).

27. Syed, S. A. \& Loesche, W. J. Survival of human dental plaque flora in various transport media. Applied microbiology 24, 638-644 (1972).

28. Caporaso, J. G. et al. Ultra-high-throughput microbial community analysis on the Illumina HiSeq and MiSeq platforms. Isme J. 6, 1621-1624 (2012).

29. Hildebrand, F., Tadeo, R., Voigt, A. Y., Bork, P. \& Raes, J. LotuS: an efficient and user-friendly OTU processing pipeline. Microbiome 2,30 (2014).

30. Edgar, R. C. UPARSE: highly accurate OTU sequences from microbial amplicon reads. Nat. Methods 10, 996-998 (2013).

31. Wang, Q., Garrity, G. M., Tiedje, J. M. \& Cole, J. R. Naive Bayesian classifier for rapid assignment of rRNA sequences into the new bacterial taxonomy. Appl. Environ Microbiol. 73, 5261-5267 (2007).

32. Caporaso, J. G. et al. QIIME allows analysis of high-throughput community sequencing data. Nat. Methods 7, 335-336 (2010).

33. Russel, J. et al. DAtest: a framework for choosing differential abundance or expression method. bioRXiv, https://doi. org/10.1101/241802 (2018).

34. Love, M. I., Huber, W. \& Anders, S. Moderated estimation of fold change and dispersion for RNA-seq data with DESeq. 2. Genome biology 15, 550 (2014).

35. Chen, X. \& Ishwaran, H. Random forests for genomic data analysis. Genomics 99, 323-329 (2012).

\section{Acknowledgements}

This study was funded with the Kootstra Talent Fellowship (Maastricht University Medical Centre). We would like to thank all patients and healthy volunteers who participated in this study. 


\section{Author contributions}

L.d.W., S.R., N.B. and J.P. designed the experiment and wrote the study protocol. L.d.W. and N.B. were involved in the selection of patients and collection of samples. J.P. performed the microarray studies. L.d.W., Z.S., S.R., M.P. and J.P. were involved in the data analysis. L.d.W., S.R., Z.S. and J.P. wrote the manuscript. All authors were involved in the interpretation of study results and reviewed the manuscript.

\section{Competing interests}

The authors declare no competing interests.

\section{Additional information}

Supplementary information is available for this paper at https://doi.org/10.1038/s41598-020-62918-8.

Correspondence and requests for materials should be addressed to Z.S.

Reprints and permissions information is available at www.nature.com/reprints.

Publisher's note Springer Nature remains neutral with regard to jurisdictional claims in published maps and institutional affiliations.

(c) (i) Open Access This article is licensed under a Creative Commons Attribution 4.0 International License, which permits use, sharing, adaptation, distribution and reproduction in any medium or format, as long as you give appropriate credit to the original author(s) and the source, provide a link to the Creative Commons license, and indicate if changes were made. The images or other third party material in this article are included in the article's Creative Commons license, unless indicated otherwise in a credit line to the material. If material is not included in the article's Creative Commons license and your intended use is not permitted by statutory regulation or exceeds the permitted use, you will need to obtain permission directly from the copyright holder. To view a copy of this license, visit http://creativecommons.org/licenses/by/4.0/.

(C) The Author(s) 2020 